\title{
Estudio de la adherencia piedra-mortero
}

The adherence in the union stone-mortar

M REYES RODRÍGUEZ GARCÍA, Dr. JUAN PEREDA MARÍN, JORGE POLO VELASCO y DR. JESÚS BARRIOS SEVILLA

\author{
RESUMEN \\ Los aplacados de piedra presentan una extensa patología \\ que se traduce en la caída de las placas colocadas. Una de \\ las causas es la falta de adherencia mortero-piedra. \\ El estudio se realiza para determinar la tensión de \\ adherencia entre diversos morteros de cemento $(1: 3,1: 5$, \\ 1:7, 1:9 y otro compuesto por mortero y látex) y piedras \\ (granito blanco, granitos rosa, granitos negros, mármol \\ blanco y calizas crema). \\ De los resultados obtenidos se deduce que los únicos \\ morteros que permiten valores de adherencia aceptables \\ (superiores a $3 \mathrm{kp} / \mathrm{cm}^{2}$ ) son el mortero de cemento $1: 3$ y el \\ especial.
}

Igualmente se comprueba que, en las piedras estudiadas, no existe relación alguna entre la adherencia y la absorción de agua.

\author{
ABSTRACT \\ Stones placates present a wide of problems that result in \\ the fall of plates. One of the causes is the lack of \\ adherence stone-mortar. \\ We considered a study to determine the adherence between \\ several cement mortars $(1: 3,1: 5,1: 7,1: 9$ and a especial \\ mortar prepared with latex) and stones (white granite, pink \\ granites, black granites, white marble and cream \\ limestones).
}

The results obtained suggest that only adecuate adherence rates (higher than $3 \mathrm{kgf} / \mathrm{cm}^{2}$ ) achieved with cement mortar 1:3 and especial mortar.

Besides it is observed that in the stones studied there is no relation between adherence and the absortion values.

\section{I.- INTRODUCCIÓN Y OBJETIVOS}

El empleo de piedra natural como revestimiento exterior ha supuesto una mejora en las características generales del edificio ya que estos materiales incrementan el aislamiento térmico y acústico además de ser más resistentes que los tradicionales ante la acción de los agentes externos, con lo que se aumenta la durabilidad de la fachada.

Esta solución se realiza, en muchos casos, por personal no cualificado y sin experiencia sobre soluciones adecuadas. Se ha podido comprobar, en varios edificios visitados en Cádiz, Huelva y Sevilla, una serie de problemas importantes cuya consecuencia más inmediata es el rechazo incipiente al uso de la piedra como aplacado. De todos los fallos y anomalías que aparecen, el de mayor entidad es la caída de placas cuyo origen es múltiple aunque siempre suele estar, como causa primera, la falta de adherencia al soporte. Hay que indicar la influencia

\section{I.- INTRODUCTION AND OBJECTIVES}

The use of natural stone as external cover has meant an improvement in the general features of buildings since natural stone increases termic and acoustic isolation and besides, it is more resistant to external agents than traditional materials increasing the durability of façades.

This solution is in many cases carried out by non qualified staff. In some buildings visited in Cadiz, Huelva and Seville some problems have been observed in the use of natural stone as placation (external covering). The most common of failures is the fall of plates which is caused in the first place by a lack of adherence to the base. It must be also noted the influence of others factors such as the contraction- expansion suffered by the materials (very important in external placations as the plates are under very high temperatures during the day and 
de otros factores como pueden ser los ciclos contracción-dilatación que sufren los materiales (muy importantes en el caso de aplacados exteriores por estar las placas sometidas a temperaturas muy elevadas durante el día disminuyendo considerablemente por la noche), la elevada humedad relativa de la ciudad en determinadas épocas y otros factores propios del material ( por ejemplo el coeficiente de absorción de agua y la compacidad).

Un breve recorrido por la normativa existente sobre este tema permite distinguir dos soluciones:

-Sistema tradicional.

-Sistema de anclajes.

Los edificios que han presentado problemas adoptaban el primero de los sistemas sin que se tenga conocimiento alguno acerca de patologías graves empleando el sistema de anclajes.

Por esta razón, ha parecido conveniente plantear un estudio de la adherencia que presentan distintos materiales pétreos a mortero, empleando diferentes dosificaciones cemento:arena con objeto de:

- Determinar los morteros adecuados para conseguir la máxima adherencia.

-Establecer una metodología de ensayo rápida y reproducible que permita prever el comportamiento de la fachada en lo que se refiere a la adherencia con los morteros.

-Comparar el comportamiento de las piedras naturales más empleadas

\section{II.- REVISIÓN DE LA NORMATIVA}

La normativa española es bastante ambigua en lo que se refiere al tipo de material de agarre y a las soluciones constructivas que permiten la sujeción de las placas a la fábrica, además de no existir ninguna norma de obligado cumplimiento.

Cabe decir que las Prescripciones Técnicas de la Dirección General de Arquitectura aconsejan el uso de morteros e indican como materiales auxiliares de unión y/o sujeción elementos tales como tornillos, escarpias y grapas de bronce o cobre y añade: “...las grapas podrán también estar formadas por alambre de hierro galvanizado o de latón de 4 ó $5 \mathrm{~mm}$ de grueso cuyos extremos deben quedar ocultos entre los calzos de las piedras...". under much lower temperatures during the night), the high relative humidity in the cities during certain seasons, and others factors inherent to the materials (the rate of water absortion and compactness).

Regarding the current regulation we can distinguish two different solutions:

-Traditional system.

-Anchorage system.

The first of the systems was used in the buildings which have shown problems. The use of the anchorage system has not known important problems. For this reason we have considered a study of the adherence between different stone materials and mortar, using different proportions of cement:sand in order to:

-Determine the correct mortar to achieve the
maximum adherence. -Establish a rapid and reproducible methodology of testing which can foresee the behaviour of façades regarding the adherence of materials when using mortars

-Compare the behaviour of the most used stones

\section{II.- REVISION OF REGULATIONS}

Spanish regulation regarding anchorage materials and building solutions which fix plates to buildings is very ambigous and besides, there is no binding regulation.

Technical Prescriptions of the Spanish Governmental Organism for Architecture advise the use of mortar and point out as auxiliary materials of union and/or fastening elements such as screws, hooks and bronze or copper cramps. This organism adds "...cramps could also be formed by galvanic iron or brass wire of 4 or $5 \mathrm{~mm}$ thickness whose ends must he hidden between the wedges of the stones...". 
Por otro lado, las Normas Tecnológicas (RPC) se decantan por las sujeciones con anclajes distinguiendo entre:

-Anclaje oculto.

-Anclaje visto.

-Anclaje de varilla.

Para los dos primeros el material debe ser acero inoxidable, especificando la NTE el límite elástico, la longitud del anclaje así como el espesor de las placas. $\mathrm{El}$ anclaje de varilla debe ser de acero $\mathrm{AE}$ de $5 \mathrm{~mm}$ de diámetro y galvanizado después de darle forma.

Ambos sistemas (anclajes y agarre con mortero) presentan una serie de inconvenientes que ocasionan, a veces, el desprendimiento o la separación de placas de la fábrica. Como causas más importantes pueden destacarse:

\section{-Sistema tradicional:}

-Inadecuada dosificación del mortero de agarre.

-Empleo de elementos auxiliares de sujeción constituidos por materiales susceptibles de corrosión. Esta cuestión entraña, además del riesgo de caída por el deterioro del elemento, otro aspecto, de carácter estético pero no menos importante, como es la mancha de herrumbre en la placa de piedra.

-Ejecución incorrecta.

El fenómeno de elementos oxidables ha ocasionado, como puede comprobarse en numerosos monumentos, el deterioro acelerado de la piedra, lo que ha supuesto el origen de patologías especialmente graves por cuanto se facilitan procesos de desagregación del material.

\section{- Sistema de anclajes:}

En principio, y si el material del que está constituido el anclaje es inoxidable, no debería ser una mala solución. Los problemas que aparecen son de naturaleza "socioeconómica" más que de índole fisicomecánica. En concreto puede ocurrir:

-Que la solución sea excesivamente cara

-Necesidad de una mano de obra cualificada en lo que se refiere a la colocación puesto que ésta suele ser complicada y determinante de la eficiencia del sistema.
Other technical regulations (RPC) are more specific with fastenings using anchorages. These regulation describe three types of anchorage:

-Hidden anchorage.

-Seen anchorage.

-Stick anchorage.

In the first two types anchorage material must be of stainless steel whose elastic limit is specified in the $N T E$, together with the lenght of the anchorage and the thickness of the plates. Stick anchorage must be of AE steel of $5 \mathrm{~mm}$ diameter and galvanized after being shaped.

Both systems anchorage and traditional (mortar) have several problems that sometimes occasionate the release or the separation of the plates from the building. The more important causes are:

\section{Traditional system:}

-Incorrect dosage of mortar.

-Use of auxiliary fastening elements subject to corrosion. Together with the risk of fall because of the damage cause in the element this problem has an aesthetic aspect, important as well. such as the stain of rust on the stone plates.

-Incorrect execution.

The existence of oxidable elements, as it is shown in many monuments has accelerated the stone deterioration, this has meant the origin of especially important problems since dissintegration processes are caused.

\section{Anchorage system:}

If the material which the anchorage is made of is stainless, this system should be a good solution. With this system appear "socio-economic" problems instead of physical-mechanical problems. It could happen that:

-The solution is very expensive.

-There is a need of qualified workers to fix the plates since it is complicated and decisive for the efficiency of the system. 
-Asimismo, control muy cuidadoso de los taladros y perforaciones de las placas de piedra, dimensiones, espesores, y demás parámetros de diseño previo fundamentales para la colocación.

Como puede deducirse de lo anteriormente expuesto, existen importantes dudas con estos sistemas. La solución adoptada en la mayoría de los proyectos realizados es una combinación de ambos, esto es, empleando anclajes fiables (suprimiendo grapas y alambres cuyo efecto dañino está más que demostrado) y mortero para el agarre de las placas a la fábrica.

Sin embargo, aun en estos casos, han ocurrido incidencias de desprendimientos de aplacados. La razón principal ha sido que se ha confiado en exceso en el anclaje y se ha descuidado el mortero, usándose dosificaciones claramente incorrectas como podrá comprobarse por los resultados obtenidos tras la realización de las experiencias objeto de este artículo.

\section{III.- METODOLOGÍA}

Se ha estudiado la resistencia a la adherencia que presenta un conjunto de rocas ornamentales ante morteros con distintas dosificaciones (1:3, 1:5, 1:7 y 1:9) y un mortero especial para este tipo de materiales, fabricado por una empresa norteamericana.

Los materiales elegidos corresponden a los tres tipos de piedra natural que se colocan normalmente en fachada, escogiéndose las más empleadas tanto en revestimientos como en zócalos de altura en torno a los dos metros. Las muestras corresponden a:

-Granitos: En total se ha dispuesto de seis muestras, tres granitos rosas, dos negros y uno blanco.

-Mármoles: Sólo se ha elegido una muestra correspondiente a mármol blanco.

-Calizas: Se han tomado dos muestras que corresponden al tipo "Caliza Crema".

Cada muestra responde a un tipo que se comercializa con distinto nombre y de cada una de ellas se han tallado quince probetas prismáticas de tres centímetros de espesor y seis centímetros de lado. Las probetas se han distribuido en grupos de tres con objeto de ensayar los diferentes morteros.

Para la realización de los ensayos se ha seguido como norma base la UNE 83.822/92 (EXPERIMENTAL) adaptándola para esta experiencia en los siguientes puntos:
-It must be taken a very careful control of the drills and perforations of the stone plates, dimension, thickness and other previous design parameters determinant in the fastening.

As it has been exposed there are several doubts about these two systems. In most of the projects a mixed solution of both has been carried out, that is, choosing reliable anchorages (supressing wires and cramps that cause damage) with mortar for the fastening of the plates to the building.

However even in the last case there has been a fall of the stoneplates. The main reason is a high reliance on the anchorage, disregarding mortar which has been clearly used in an incorrect dosage as it is shown in the results obtained in the study described among these lines.

\section{III.- METHODOLOGY}

It has been studied the adherence of a group of ornamental rocks to mortar of different proportion between cement:sand $(1: 3,1: 5,1: 7,1: 9)$ and to an especial mortar for these rocks manufacture in USA.

The selected materials belong to the three types of natural stones that are normally placed in façades, and the most commonly used in wall coverings and high socles (aproximately $2 \mathrm{~m}$ ) were chosen. The samples were:

-Granites: Six samples, three pink granites, two black granites and one white granite.

-Marbles: One sample of white marble.

-Limestones: Two samples of "Cream limestone".

Each sample corresponds to a type that is commercialised with a different name. Of every sample, 15 prismatic testers of $3 \mathrm{~cm}$ thickness and $6 \mathrm{~cm}$ lenght have been cut. The testers have been distributed in groups of three in order to test all the different mortars

The tests have been carried out according to the basic regulation UNE 83.822/92 (EXPERIMENTAL) but changing it for this study in the following points: 
-En el apartado "PREPARACIÓN Y COLOCACIÓN DE LAS PROBETAS" se ha optado por situarlas a la intemperie con objeto de que sufran las mismas inclemencias climáticas que en obra.

-No se ha procedido al corte de la probeta a la hora de realizar la medida ya que esta operación alteraría los resultados en morteros con suficiente adherencia o bien anularía la medida en aquellos morteros con baja adherencia.

-El soporte ha sido una placa de hormigón en el caso del mortero especial; en el resto se ha colocado el mortero sobre plástico con idea de obtener la medida de la adherencia piedra-mortero sin que ocurran interferencias.

Para la ejecución del ensayo se ha empleado un dinamómetro que permite la obtención de datos desde 0 a 500 kilopondios. Las placas de estiramiento son pastillas de acero que se adhieren a las piedras con un pegamento adecuado.

También se ha medido el coeficiente de absorción de agua de cada material ensayado según UNE 22-17285.

En la foto 1 se muestran las probetas colocadas con el montaje previo al ensayo.
-In the paragraph "SET OUT' AND FIXING OF THE TESTERS" the testers have been situated outdoors in order that they suffer the same atmospheric conditions than in building site.

-Testers have not been cut when evaluating the test as cutting them would have altered the results in mortars with sufficient adherence or it would have supressed the evaluation in those mortars of less adherence.

-The base has been a concrete plate when using the special mortar; For the restant mortars a plastic base has been used in order to evaluate the adherence stone-mortar without interferences.

For carrying out the study a dynamometer with a capacity of 0 to $500 \mathrm{kgF}$ has been used. The stretching plaques are steel pieces that are fixed to the testers with an appropiate glue.

The rate of water absortion has also been measured in every material tested according to UNE 22-17285.

The photo I shows the testers before the test was made.

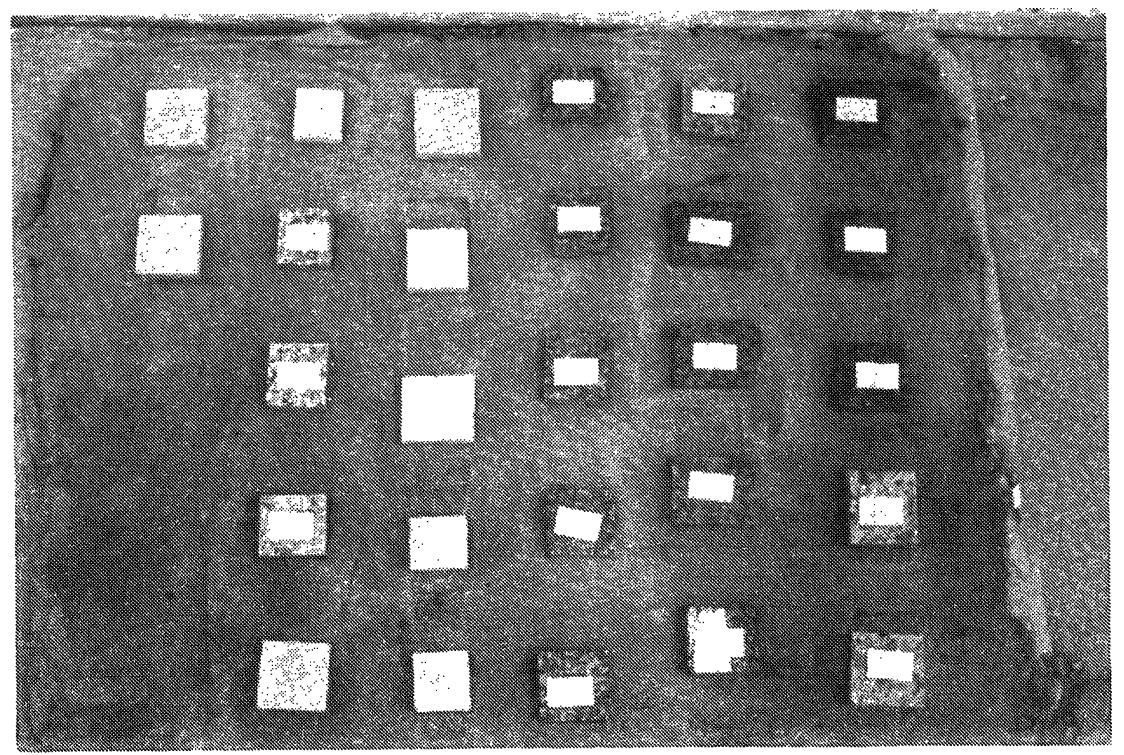

Foto 1 (Photo 1)

\section{IV.- RESULTADOS}

A los 28 días de haberse efectuado la unión piedramortero, se procedió a la ejecución de los ensayos de adherencia obteniéndose los siguientes resultados.

\section{IV.- RESULTS}

After 28 days of the union stone-mortar, the test of adherence was carried out. The results were the following: 
GRANITOS (GRANITES)

1.- GRANITO BLANCO (WHITE GRANITE):

\begin{tabular}{|c|c|c|c|c|}
\hline Mortero & Probeta & $\begin{array}{l}\text { Superficie } \\
\qquad \mathrm{cm}^{2}\end{array}$ & $\begin{array}{l}\text { Lectura } \\
\text { dinamómetro } \\
\text { (kp) }\end{array}$ & $\begin{array}{l}\text { Adherencia } \\
\left(\mathrm{kp} / \mathrm{cm}^{2}\right)\end{array}$ \\
\hline \multirow{3}{*}{$1: 3$} & 1 & 23,92 & 115 & 4,81 \\
\hline & 2 & 29,72 & 129 & 4,36 \\
\hline & 3 & 26,15 & 118 & 4,51 \\
\hline \multirow{3}{*}{$1: 5$} & 4 & 25,19 & 57 & 2,26 \\
\hline & 5 & 27,32 & 63 & 2,31 \\
\hline & 6 & 24,34 & 60 & 2,48 \\
\hline \multirow{3}{*}{$1: 7$} & 7 & 24,02 & 28 & 1,17 \\
\hline & 8 & 31,95 & 33 & 1,03 \\
\hline & 9 & 31,18 & 35 & 1,12 \\
\hline \multirow{3}{*}{$1: 9$} & 10 & 31,49 & 17 & 0,54 \\
\hline & 11 & 32,48 & 18 & 0,57 \\
\hline & 12 & 25,93 & 13 & 0,50 \\
\hline \multirow{3}{*}{ ESPECIAL } & 13 & 31,83 & 299 & 9,39 \\
\hline & 14 & 31,38 & 325 & 10,36 \\
\hline & 15 & 32,21 & 295 & 9,16 \\
\hline
\end{tabular}

2.- GRANITOS ROSAS (PINK GRANITES)

2.1.- Granito rosa tipo 1 (Pink granite type 1):

\begin{tabular}{|c|c|c|c|c|}
\hline Mortero & Probeta & $\begin{array}{c}\text { Superficie } \\
\mathrm{cm}^{2}\end{array}$ & $\begin{array}{l}\text { Lectura } \\
\text { dinamómetro } \\
(k p)\end{array}$ & $\begin{array}{l}\text { Adherencia } \\
\left(\mathrm{kp} / \mathrm{cm}^{2}\right)\end{array}$ \\
\hline \multirow{3}{*}{$1: 3$} & 1 & 39,28 & 155 & 3,95 \\
\hline & 2 & 34,52 & 145 & 4,21 \\
\hline & 3 & 34,15 & 133 & 3,89 \\
\hline \multirow{3}{*}{$1: 5$} & 4 & 33,21 & 77 & 2,32 \\
\hline & 5 & 34,30 & 97 & 2,83 \\
\hline & 6 & 38,97 & 88 & 2,26 \\
\hline \multirow{3}{*}{$1: 7$} & 7 & 39,61 & 72 & 1,82 \\
\hline & 8 & 38,88 & 72 & 1,85 \\
\hline & 9 & 33,94 & 66 & 1,95 \\
\hline \multirow{3}{*}{$1: 9$} & 10 & 41,26 & 31 & 0,75 \\
\hline & 11 & 39,87 & 36 & 0,92 \\
\hline & 12 & 33,59 & 30 & 0,89 \\
\hline \multirow{3}{*}{ ESPECIAL } & 13 & 37,02 & 202 & 5,46 \\
\hline & 14 & 31,65 & 162 & 5,12 \\
\hline & 15 & 33,86 & 193 & 5,70 \\
\hline
\end{tabular}


2.2.- Granito rosa tipo 2 (Pink granite type 2):

\begin{tabular}{|c|c|c|c|c|}
\hline Mortero & Probeta & $\begin{array}{c}\text { Superficie } \\
\mathrm{cm}^{2}\end{array}$ & $\begin{array}{c}\text { Lectura } \\
\text { dinamómetro } \\
\text { (kp) }\end{array}$ & $\begin{array}{l}\text { Adherencia } \\
\left(\mathbf{k p} / \mathrm{cm}^{2}\right)\end{array}$ \\
\hline \multirow{3}{*}{$1: 3$} & 1 & 41,89 & 86 & 2,06 \\
\hline & 2 & 45,72 & 90 & 1,98 \\
\hline & 3 & 45,26 & 84 & 1,86 \\
\hline \multirow{3}{*}{$1: 5$} & 4 & 47,81 & 66 & 1,38 \\
\hline & 5 & 46,61 & 74 & 1,59 \\
\hline & 6 & 44,90 & 65 & 1,45 \\
\hline \multirow{3}{*}{$1: 7$} & 7 & 44,43 & 53 & 1,19 \\
\hline & 8 & 44,82 & 55 & 1,23 \\
\hline & 9 & 45,60 & 62 & 1,36 \\
\hline \multirow{3}{*}{$1: 9$} & 10 & 44,55 & 34 & 0,77 \\
\hline & 11 & 45,21 & 29 & 0,65 \\
\hline & 12 & 44,75 & 21 & 0,47 \\
\hline \multirow{3}{*}{ ESPECIAL } & 13 & 46,13 & 334 & 7,25 \\
\hline & 14 & 45,38 & 311 & 6,86 \\
\hline & 15 & 44,16 & 340 & 7,70 \\
\hline
\end{tabular}

2.3.- Granito rosa tipo 3 (Pink granite type 3):

\begin{tabular}{|c|c|c|c|c|}
\hline Mortero & Probeta & $\begin{array}{l}\text { Superficie } \\
\mathrm{cm}^{2}\end{array}$ & $\begin{array}{c}\text { Lectura } \\
\text { dinamómetro } \\
(k p)\end{array}$ & $\begin{array}{l}\text { Adherencia } \\
\left(\mathrm{kp} / \mathrm{cm}^{2}\right)\end{array}$ \\
\hline \multirow{3}{*}{$1: 3$} & 1 & 36,73 & 103 & 2,80 \\
\hline & 2 & 36,52 & 110 & 3,01 \\
\hline & 3 & 35,13 & 99 & 2,82 \\
\hline \multirow{3}{*}{$1: 5$} & 4 & 36,27 & 48 & 1,32 \\
\hline & 5 & 34,01 & 48 & 1,41 \\
\hline & 6 & 36,80 & 52 & 1,41 \\
\hline \multirow{3}{*}{$1: 7$} & 7 & 37,29 & 28 & 0,75 \\
\hline & 8 & 35,07 & 34 & 0,97 \\
\hline & 9 & 37,74 & 41 & 1,09 \\
\hline \multirow{3}{*}{$1: 9$} & 10 & 36,83 & 13 & 0,35 \\
\hline & 11 & 35,82 & 23 & 0,64 \\
\hline & 12 & 34,22 & 19 & 0,55 \\
\hline \multirow{3}{*}{ ESPECIAL } & 13 & 36,61 & 87 & 2,38 \\
\hline & 14 & 33,08 & 80 & 2,42 \\
\hline & 15 & 34,45 & 73 & 2,12 \\
\hline
\end{tabular}


3.- GRANITOS NEGROS (BLACK GRANITES)

3.1.- Granito negro tipo 1 (Black granite type 1):

\begin{tabular}{|c|c|c|c|c|}
\hline Mortero & Probeta & $\begin{array}{c}\text { Superficie } \\
\mathrm{cm}^{2}\end{array}$ & $\begin{array}{l}\text { Lectura } \\
\text { dinamómetro } \\
(\mathbf{k p})\end{array}$ & $\begin{array}{l}\text { Adherencia } \\
\left(\mathrm{kp} / \mathrm{cm}^{2}\right)\end{array}$ \\
\hline \multirow{3}{*}{$1: 3$} & 1 & 34,64 & 108 & 3,12 \\
\hline & 2 & 34,96 & 120 & 3,43 \\
\hline & 3 & 34,63 & 107 & 3,09 \\
\hline \multirow{3}{*}{$1: 5$} & 4 & 34,47 & 75 & 2,16 \\
\hline & 5 & 34,93 & 68 & 1,94 \\
\hline & 6 & 34,08 & 72 & 2,12 \\
\hline \multirow{3}{*}{$1: 7$} & 7 & 34,61 & 47 & 1,36 \\
\hline & 8 & 35,44 & 63 & 1,78 \\
\hline & 9 & 24,48 & 60 & 1,74 \\
\hline \multirow{3}{*}{$1: 9$} & 10 & 34,84 & 22 & 0,63 \\
\hline & 11 & 34,64 & 17 & 0,50 \\
\hline & 12 & 35,13 & 21 & 0,60 \\
\hline \multirow{3}{*}{ ESPECIAL } & 13 & 35,57 & 202 & 5,67 \\
\hline & 14 & 17,78 & 113 & 6,37 \\
\hline & 15 & 20,28 & 122 & 6,02 \\
\hline
\end{tabular}

3.2.- Granito negro tipo 2 (Black granite type 2):

\begin{tabular}{|c|c|c|c|c|}
\hline Mortero & Probeta & $\begin{array}{l}\text { Superficie } \\
\mathrm{cm}^{2}\end{array}$ & $\begin{array}{c}\text { Lectura } \\
\text { dinamómetro } \\
\text { (kp) }\end{array}$ & $\begin{array}{l}\text { Adherencia } \\
\left(\mathrm{kp} / \mathrm{cm}^{2}\right)\end{array}$ \\
\hline \multirow{3}{*}{$1: 3$} & 1 & 34,24 & 84 & 2,45 \\
\hline & 2 & 30,72 & 85 & 2,77 \\
\hline & 3 & 34,00 & 88 & 2,59 \\
\hline \multirow{3}{*}{$1: 5$} & 4 & 33,01 & 47 & 1,42 \\
\hline & 5 & 33,04 & 48 & 1,45 \\
\hline & 6 & 31,92 & 49 & 1,53 \\
\hline \multirow{3}{*}{$1: 7$} & 7 & 30,47 & 39 & 1,28 \\
\hline & 8 & 35,59 & 40 & 1,12 \\
\hline & 9 & 36,88 & 49 & 1,33 \\
\hline \multirow[b]{3}{*}{$1: 9$} & 10 & 36,58 & 12 & 0,33 \\
\hline & 11 & 36,38 & 20 & 0,55 \\
\hline & 12 & 34,94 & 15 & 0,43 \\
\hline \multirow{3}{*}{ ESPECIAL } & 13 & 35,22 & 421 & 11,95 \\
\hline & 14 & 33,75 & 420 & 12,44 \\
\hline & 15 & 35,39 & 360 & 10,17 \\
\hline
\end{tabular}


MÁRMOLES (MARBLES)

MÁRMOL BLANCO (WHITE MARBLE):

\begin{tabular}{|c|c|c|c|c|}
\hline Mortero & Probeta & $\begin{array}{l}\text { Superficie } \\
\mathrm{cm}^{2}\end{array}$ & $\begin{array}{c}\text { Lectura } \\
\text { dinamómetro } \\
\text { (kp) }\end{array}$ & $\begin{array}{l}\text { Adherencia } \\
\left(\mathrm{kp} / \mathrm{cm}^{2}\right)\end{array}$ \\
\hline \multirow{3}{*}{$1: 3$} & 1 & 23,06 & 56 & 2,43 \\
\hline & 2 & 23,47 & 63 & 2,68 \\
\hline & 3 & 22,98 & 52 & 2,25 \\
\hline \multirow{3}{*}{$1: 5$} & 4 & 25,43 & NULA & - \\
\hline & 5 & 25,13 & 52 & 2,07 \\
\hline & 6 & 22,33 & 53 & 2,37 \\
\hline \multirow{3}{*}{$1: 7$} & 7 & 22,16 & 34 & 1,53 \\
\hline & 8 & 22,33 & 39 & 1,75 \\
\hline & 9 & 21,71 & 36 & 1,66 \\
\hline \multirow{3}{*}{$1: 9$} & 10 & 22,76 & 16 & 0,72 \\
\hline & 11 & 23,00 & 23 & 0,99 \\
\hline & 12 & 24,10 & 23 & 0,97 \\
\hline \multirow{3}{*}{ ESPECIAL } & 13 & 22,32 & 262 & 11,73 \\
\hline & 14 & 21,60 & 240 & 11,11 \\
\hline & 15 & 25,40 & 323 & 12,71 \\
\hline
\end{tabular}

CALIZAS (LIMESTONES)

1.- CALIZA TIPO 1 (LIMESTONE TYPE 1):

\begin{tabular}{|c|c|c|c|c|}
\hline Mortero & Probeta & $\begin{array}{c}\text { Superficie } \\
\mathrm{cm}^{2}\end{array}$ & $\begin{array}{c}\text { Lectura } \\
\text { dinamómetro } \\
\text { (kp) }\end{array}$ & $\begin{array}{c}\text { Adherencia } \\
\left(\mathrm{kp} / \mathrm{cm}^{2}\right)\end{array}$ \\
\hline \multirow{3}{*}{$1: 3$} & 1 & 37,77 & 80 & 2,12 \\
\hline & 2 & 35,76 & 70 & 1,96 \\
\hline & 3 & 35,25 & 78 & 2,21 \\
\hline \multirow{3}{*}{$1: 5$} & 4 & 35,97 & 40 & 1,11 \\
\hline & 5 & 35,41 & 46 & 1,30 \\
\hline & 6 & 34,69 & 50 & 1,44 \\
\hline \multirow{3}{*}{$1: 7$} & 7 & 34,92 & 22 & 0,63 \\
\hline & 8 & 34,52 & 24 & 0,69 \\
\hline & 9 & 34,76 & 25 & 0,72 \\
\hline \multirow{3}{*}{$1: 9$} & 10 & 34,73 & 9 & 0,26 \\
\hline & 11 & 35,31 & 13 & 0,37 \\
\hline & 12 & 35,20 & 10 & 0,28 \\
\hline \multirow{3}{*}{ ESPECIAL } & 13 & 34,40 & 384 & 11,16 \\
\hline & 14 & 34,46 & 425 & 12,34 \\
\hline & 15 & 34,82 & 386 & 11,09 \\
\hline
\end{tabular}


2.- CALIZA TIPO 2 (LIMESTONE TYPE 2):

\begin{tabular}{|c|c|c|c|c|}
\hline Mortero & Probeta & $\begin{array}{c}\text { Superficie } \\
\mathrm{cm}^{2}\end{array}$ & $\begin{array}{c}\text { Lectura } \\
\text { dinamómetro } \\
\text { (kp) }\end{array}$ & $\begin{array}{c}\text { Adherencia } \\
\left(\mathrm{kp} / \mathrm{cm}^{2}\right)\end{array}$ \\
\hline \multirow{3}{*}{$1: 3$} & 1 & 35,67 & 159 & 4,47 \\
\hline & 2 & 37,00 & 174 & 4,70 \\
\hline & 3 & 35,31 & 158 & 4,49 \\
\hline \multirow{3}{*}{$1: 5$} & 4 & 38,68 & 59 & 1,52 \\
\hline & 5 & 36,13 & 47 & 1,30 \\
\hline & 6 & 37,92 & 58 & 1,53 \\
\hline \multirow{3}{*}{$1: 7$} & 7 & 36,89 & 50 & 1,35 \\
\hline & 8 & 40,27 & 48 & 1,19 \\
\hline & 9 & 41,17 & 61 & 1,48 \\
\hline \multirow{3}{*}{$1: 9$} & 10 & 36,13 & 17 & 0,47 \\
\hline & 11 & 37,92 & 20 & 0,53 \\
\hline & 12 & 44,96 & 18 & 0,40 \\
\hline \multirow{3}{*}{ ESPECIAL } & 13 & 44,16 & 463 & 10,48 \\
\hline & 14 & 36,90 & 420 & 11,38 \\
\hline & 15 & 41,02 & 416 & 10,14 \\
\hline
\end{tabular}

En las tablas y gráficos siguientes se muestran los valores medios de adherencia obtenidos para cada material y mortero correspondiente:
The following tables and diagrams show the adherence mean rates obtained for each material and the mortar used with it:

TABLA 1.- Valores adherencia $\left(\mathrm{kp} / \mathrm{cm}^{2}\right)$ (granitos)

TABLE 1.- Adherence rates $\left(\mathrm{kg} / \mathrm{cm}^{2}\right)$ (granites)

\begin{tabular}{|c|c|c|c|c|c|c|}
\hline \multicolumn{2}{|c|}{ Material } & $\begin{array}{c}\text { Mortero } \\
1: 3\end{array}$ & $\begin{array}{c}\text { Mortero } \\
1: 5\end{array}$ & $\begin{array}{c}\text { Mortero } \\
1: 7\end{array}$ & $\begin{array}{c}\text { Mortero } \\
1: 9\end{array}$ & $\begin{array}{c}\text { Mortero } \\
\text { especial }\end{array}$ \\
\hline \multirow{2}{*}{ BLANCO } & 4,56 & 2,35 & 1,11 & 0,54 & 9,64 \\
\hline \multirow{3}{*}{ ROSAS } & TIPO 1 & 4,02 & 2,47 & 1,87 & 0,85 & 5,43 \\
\cline { 2 - 7 } & TIPO 2 & 1,97 & 1,47 & 1,26 & 0,63 & 7,27 \\
\cline { 2 - 7 } & TIPO 3 & 2,88 & 1,38 & 0,94 & 0,51 & 2,31 \\
\hline \multirow{3}{*}{ NEGROS } & TIPO 1 & 3,21 & 2,07 & 1,63 & 0,58 & 6,02 \\
\cline { 2 - 8 } & TIPO 2 & 2,60 & 1,47 & 1,24 & 0,44 & 11,52 \\
\hline
\end{tabular}


TABLA II.- Valores medios adherencia $\left(\mathrm{kp} / \mathrm{cm}^{2}\right)$ (mármoles y calizas)

TABLE II.- Adherence mean rates $\left(\mathrm{kgf} / \mathrm{cm}^{2}\right)$ (marbles and limestones)

\begin{tabular}{|c|c|c|c|c|c|c|}
\hline \multicolumn{2}{|c|}{ Material } & $1: 3$ & $1: 5$ & $1: 7$ & $1: 9$ & Especial \\
\hline \multicolumn{2}{|c|}{ MÁRMOLES } & 2,45 & 2,22 & 1,65 & 0,89 & 11,85 \\
\hline \multirow{2}{*}{ CALIZAS } & TIPO 1 & 2,10 & 1,28 & 0,68 & 0,30 & 11,53 \\
\cline { 2 - 7 } & TIPO2 & 4,54 & 1,45 & 1,34 & 0,47 & 10,67 \\
\hline
\end{tabular}

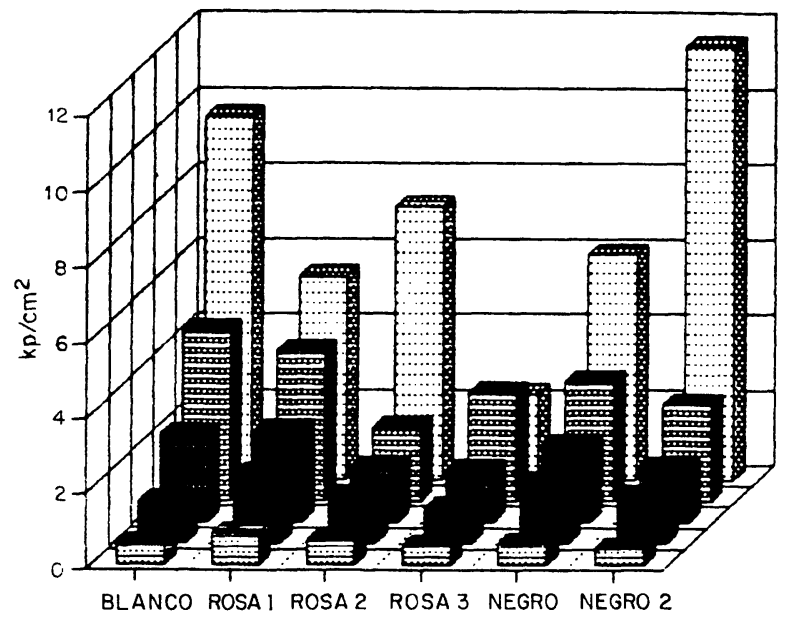

ESPECIAL 1:30 1:50 1:70 1:90

Gráfico I.- Granitos

Diagram I.- Granite.

En la tabla siguiente se recogen los datos obtenidos para los morteros 1:3 y especial, así como los valores de compacidad y coeficiente de absorción para cada material

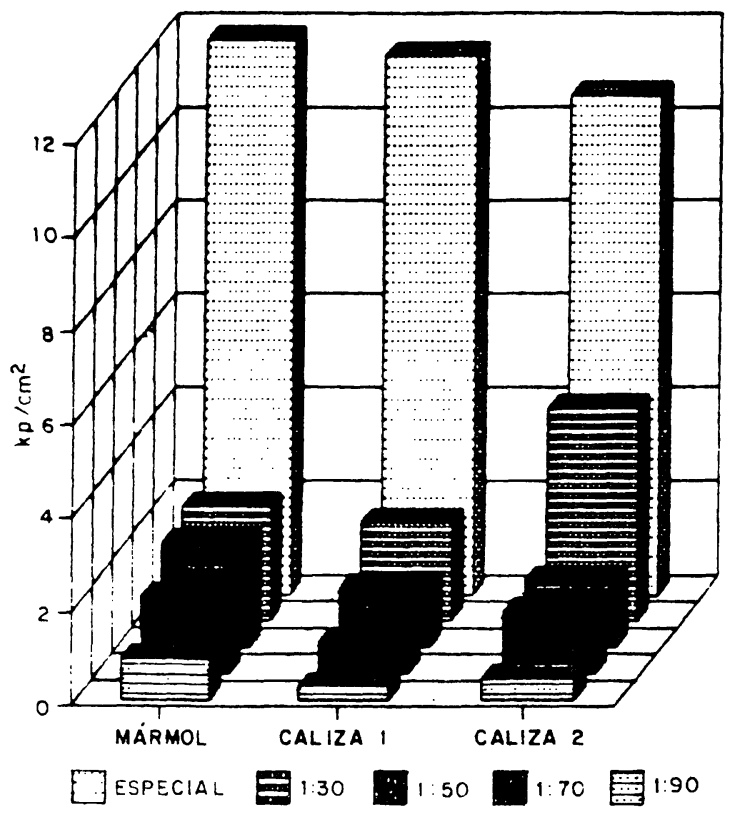

Gráfico II.- Mármoles y calizas

Diagram II.- Marbles and limestones

The following table shows the results obtained with mortars 1:3 and especial, compactness and absortion rate of each material are also shown.

\begin{tabular}{|c|c|c|c|c|}
\hline Material & $\begin{array}{c}\text { Coeficiente } \\
\text { de absorcion } \\
(\%)\end{array}$ & $\begin{array}{c}\text { Compacidad } \\
(\%)\end{array}$ & $\begin{array}{c}\text { Adherencia } \\
\text { mortero 1:3 } \\
\left(\mathbf{k p} / \mathrm{cm}^{2}\right)\end{array}$ & $\begin{array}{c}\text { Adherencia } \\
\text { mortero } \\
\text { especial }\left(\mathrm{kp}_{\mathrm{cm}} \mathbf{c m}^{2}\right)\end{array}$ \\
\hline G. BLANCO & 0,31 & 99,16 & 4,56 & 9,64 \\
\hline G. ROSA TIPO 1 & 0,33 & 99,14 & 4,02 & 5,43 \\
\hline G. ROSA TIPO 2 & 0,16 & 99,57 & 1,97 & 7,27 \\
\hline G. ROSA TIPO 3 & 0,15 & 99,60 & 2,88 & 2,31 \\
\hline $\begin{array}{c}\text { G. NEGRO } \\
\text { TIPO 1 }\end{array}$ & 0,11 & 99,69 & 3,21 & 6,02 \\
\hline $\begin{array}{c}\text { G. NEGRO } \\
\text { TIPO 2 }\end{array}$ & 0,17 & 99,52 & 2,60 & 11,52 \\
\hline $\begin{array}{c}\text { MARMOL } \\
\text { BLANCO }\end{array}$ & 0,10 & 99,70 & 2,45 & 11,85 \\
\hline CALIZA TIPO 1 & 1,72 & 95,59 & 2,10 & 11,53 \\
\hline CALIZA TIPO 2 & 0,42 & 98,87 & 4,54 & 10,67 \\
\hline
\end{tabular}


Las fotos 2 a 5 muestran algunas de las probetas ensayadas; la 2 y 3 corresponden al mortero de cemento con el que las piedras han presentado mejores adherencias la 4 y 5 son representativas de lo ocurrido con el mortero 1:9

\section{Mortero 1:3.- Obsérvese parte del mortero} adherido a la probeta.

Mortar 1:3.- Part of the mortar is stuck on the tester.

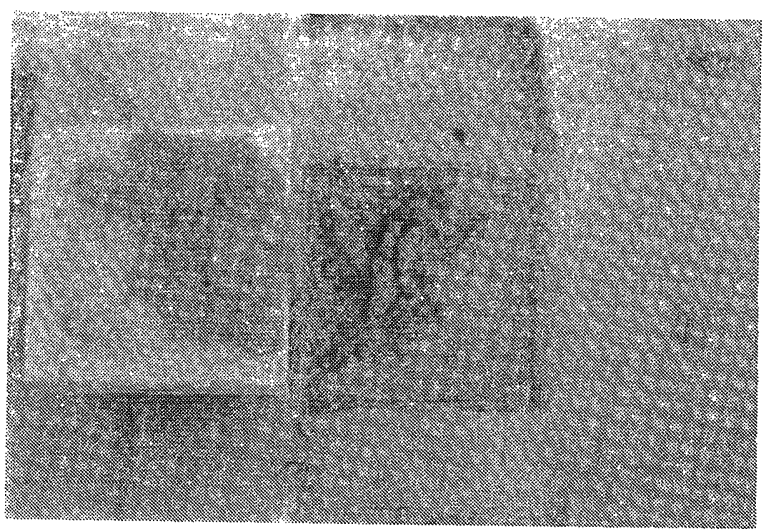

Foto 2 (Photo 2)

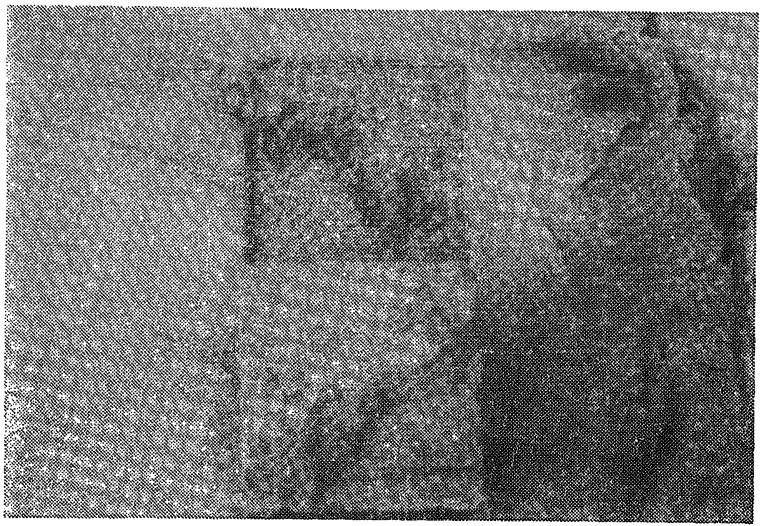

Foto 3 (Photo 3)

\section{V.- CONSIDERACIONES FINALES}

Los aplacados de piedra presentan una extensa patología que se traduce en la caída de las piezas colocadas. Las causas son diversas estando relacionadas, fundamentalmente, con el tipo de agarre o sujeción, disposición de juntas de dilatación, adherencia de los morteros empleados, corrosión del material utilizado como anclaje, ejecución incorrecta,...
The 2 to 5 photographs show some of the testers. In the 2 and 3 of them appears the cement mortar with which the stones have proved better adherence. The 4 and 5 photographs correspond to the test with mortar 1:9

Mortero 1:9.- Nótese que la piedra aparece limpia de mortero.

Mortar 1:9.- See that the stone is clean of mortar.

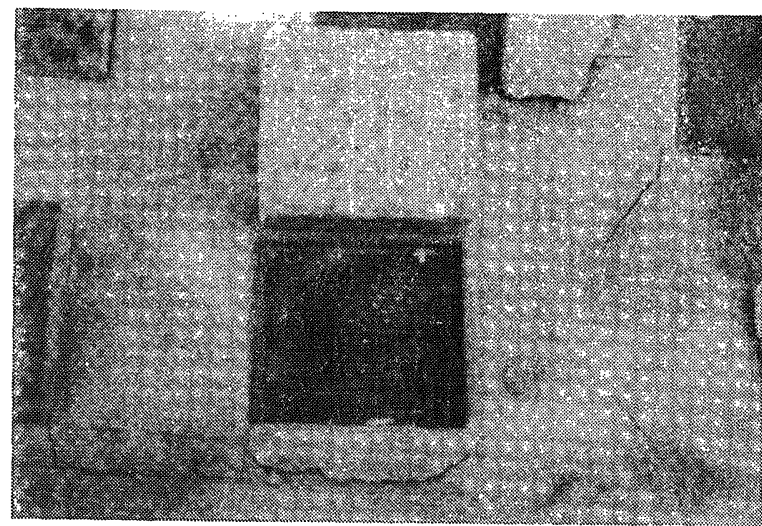

Foto 4 (Photo 4)

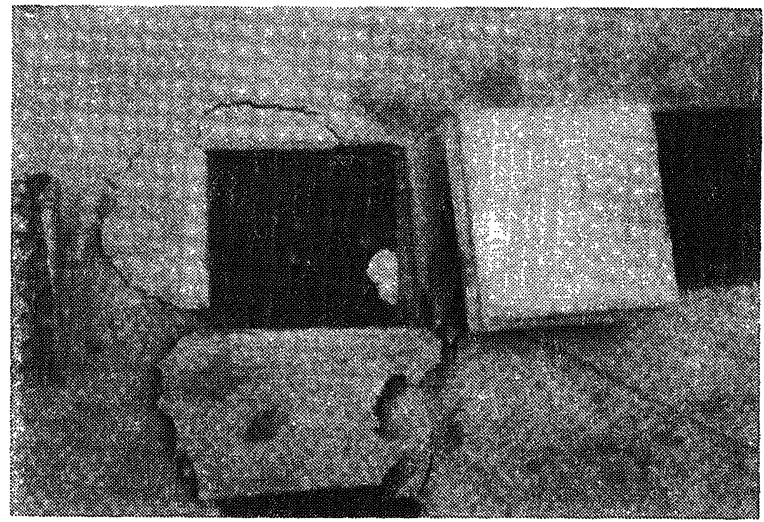

Foto 5 (Photo 5)

\section{V.- FINAL EVALUATION}

Stone placates present a wide range of problems that result in the fall of plates. Causes are different and the are mainly related to the type of fastening, location of the expansion seam, adherence of mortar. anchorage corrosion, incorrect execution... 
En este trabajo se procede al estudio de la adherencia de diversas piedras al mortero. Para ello se han elegido tres tipos distintos de granitos rosas, dos granitos negros y otro blanco; una muestra de mármol, tipo Macael, y dos de caliza crema.

Los morteros de agarre empleados están confeccionados a base de cemento y arena con dosificaciones en volumen $1: 3,1: 5,1: 7$ y 1:9 y un mortero especial preparado, según las instrucciones del fabricante, mezclando el mortero y el aditivo látex, sin adición de agua, suministrados por el mismo.

Las probetas de piedra se cogieron con cada uno de estos morteros sobre un soporte de hormigón, en unos casos, y de plástico en otros. La conservación ha sido a la intemperie con temperaturas que han oscilado entre 13 y $27^{\circ} \mathrm{C}$ y humedad del aire entre 57 y $86 \%$.

A los 28 días se procedió a realizar el ensayo de adherencia según UNE 83.822/92.

Los resultados obtenidos indican:

-Con el granito blanco se consiguen adherencias superiores a $3 \mathrm{~kg} / \mathrm{cm}^{2}$ empleando el mortero $1: 3(4,56$ $\mathrm{kg} / \mathrm{cm}^{2}$ ) media de tres ensayos) y con el mortero especial $\left(9,64 \mathrm{~kg} / \mathrm{cm}^{2}\right.$ media de tres ensayos).

-De los granitos rosas, sólo el tipo 1 da valores de adherencia superiores a $3 \mathrm{~kg} / \mathrm{cm}^{2}$ con un mortero $1: 3$ $\left(4,02 \mathrm{~kg} / \mathrm{cm}^{2}\right.$ media de tres ensayos). Con el mortero especial, las piedras tipo 1 y tipo 2 dan valores de 5,43 y $7,27 \mathrm{~kg} / \mathrm{cm}^{2}$ respectivamente en tanto que el tipo 3 da un valor de 2,31, inferior a los $3 \mathrm{~kg} / \mathrm{cm}^{2}$. Por ello puede decirse que la muestra tipo 3 no debe colocarse, ni con mortero de cemento, ni con mortero especial.

-El granito negro tipo 1, tomado con mortero 1:3 tiene una adherencia de $3,21 \mathrm{~kg} / \mathrm{cm}^{2}$. Con los demás morteros de cemento da valores inferiores a $3 \mathrm{~kg} / \mathrm{cm}^{2}$, mientras que con el mortero especial da una adherencia de $6,02 \mathrm{~kg} / \mathrm{cm}^{2}$.

Sin embargo, el granito negro tipo 2 sólo da valores de adherencia superior a $3 \mathrm{~kg} / \mathrm{cm}^{2}$ cuando se emplea el mortero especial $\left(11,52 \mathrm{~kg} / \mathrm{cm}^{2}\right)$.

-El mármol tipo Macael proporciona valores de adherencia superiores a $3 \mathrm{~kg} / \mathrm{cm}^{2}$ cuando se emplea el mortero especial $\left(11,85 \mathrm{~kg} / \mathrm{cm}^{2}\right)$.

-La piedra caliza tipo 1 sólo tiene una adherencia superior a $3 \mathrm{~kg} / \mathrm{cm}^{2}$ cuando se toma con mortero especial $(11,53)$. La caliza tipo 2 supera dicho valor
In this study the adherence of different stones to mortar has been studied. The rocks selected were: three different types of pink granite, two types of black granites and one white granite; one sample of white marble (Macael type) and two samples of cream limestones.

The mortars used were made of cement and sand with proportions of 1:3,1:5,1:7,1:9 and a especial mortar from USA prepared mixing mortar with latex without water.

The stone testers were fixed with each mortar to a base of concrete in some cases, and to a base of plastic in other cases. The test was outdoors with temperatures ranging from 13 to $27^{\circ} \mathrm{C}$ and a relative humidity between 57 and $86 \%$.

After 28 days the test was carried out according to UNE 83.822/92.

The results obtained suggest that:

-With white granite, adherences higher than $3 \mathrm{~kg} /$ $\mathrm{cm}^{2}$ are obtained when using mortar $1: 3(4,56 \mathrm{~kg} f$ $\mathrm{c}^{2} \mathrm{~m}^{2}$, mean of the test).

-Only with pink granite type 1 adherence is higher than $3 \mathrm{~kg} / \mathrm{cm}^{2}$ with mortar $1: 3\left(4,02 \mathrm{~kg} / / \mathrm{cm}^{2}\right.$, mean of three tests). With the especial mortar type 1 and type 2 of pink granite give rates of adherence of 5.43 and $7.27 \mathrm{~kg} / \mathrm{cm}^{2}$ respectively, where as type 3 gives an adherence rates of 2,31 , less than $3 \mathrm{kgf} / \mathrm{cm}^{2}$. Type 3 of pink granite should not be fixed with cement mortar nor with especial mortar.

-Black granite type 1 fixed with mortar 1:3 has an adherence rate of $3,21 \mathrm{~kg} / \mathrm{cm}^{2}$. With other cement mortars lesser adherence rates than $3 \mathrm{~kg} / \mathrm{cm} \mathrm{cm}^{2}$ are obtained, whereas with the especial mortar an adherence rate of $6,02 \mathrm{~kg} f / \mathrm{cm}^{2}$ is obtained. However. black granite type 2 gives an adherence rate higher than $3 \mathrm{~kg} f / \mathrm{cm}^{2}$ when using the especial mortar $(11,52$ $\mathrm{kg} f\left(\mathrm{~cm}^{2}\right)$.

-With white marble (Macael type) an adherence rate higher than $3 \mathrm{~kg} / \mathrm{cm}^{2}$ is obtained when using the especial mortar $\left(11,85 \mathrm{~kg} f / \mathrm{cm}^{2}\right)$.

-Limestone type 1 has only an adherence higher than $3 \mathrm{kgf} / \mathrm{cm}^{2}$ when using the especial mortar $(11,53)$. Limestone type 2 only surpasses $3 \mathrm{kgf} / \mathrm{cm}^{2}$ when 
con el mortero de cemento $1: 3\left(4,54 \mathrm{~kg} / \mathrm{cm}^{2}\right)$ y con el mortero especial $\left(10,67 \mathrm{~kg} / \mathrm{cm}^{2}\right)$.

-No existe correspondencia entre los valores de absorción de los diferentes tipos de piedra y la adherencia con los diferentes tipos de mortero empleados. Incluso en el mismo tipo de piedra, no se puede relacionar mayor coeficiente de absorciónmayor adherencia ni al contrario.

\section{VI.- CONCLUSIONES}

Tras la realización del ensayo y a la vista de los resultados obtenidos, puede concluirse:

-Si el agarre de la piedra a la fábrica se realiza con mortero de cemento, como es el caso de los aplacados interiores, el tipo recomendado es el de dosificación $1: 3$ si se quieren conseguir adherencias superiores a 3 $\mathrm{kg} / \mathrm{cm}^{2}$, que se toma como valor mínimo admisible para tener seguridad en el agarre.

-Empleando morteros especiales ( mezcla de mortero y aditivo látex) se alcanzan valores de adherencia muy altos en todos los materiales ensayados, exceptuando el granito rosa tipo 3.

-Los datos obtenidos no permiten distinguir qué tipo de piedra se comporta mejor, por lo que se recomienda la realización de este ensayo en cada caso particular.

-Asimismo, no se encuentra relación alguna entre la adherencia y la absorción de las piedras ensayadas.

\section{AGRADECIMIENTOS}

Los autores desean expresar su agradecimiento a la Empresa VORSEVI, S.A. sin cuya colaboración no habría sido posible la realización de este trabajo, así como al Ministerio de Educación y Ciencia por la ayuda concedida para el Intercambio de Personal Investigador entre Industrias y Centros Públicos de Investigación. using cement mortar $1: 3\left(4,54 \mathrm{kgf} / \mathrm{cm}^{2}\right)$ and the especial mortar $\left(10,67 \mathrm{~kg} / \mathrm{cm}^{2}\right)$.

-There is no relation between the absortion values of the different types of stones and the different types of mortar used. Even with the same type of stone the relation higher absortion-higher adherence cannot be established.

\section{VI.- CONCLUSIONS}

After the test has been carried out and the results evaluated, it can be concluded that:

-If the fastening of the stone to the building is cement mortar, the type recommended is the proportion 1:3 in order to achieve adherences higher than $3 \mathrm{kgf \textrm {cm } ^ { 2 }}$. being this value the minimum to ensure the fastering.

- Using special mortars (a mixture of latex and mortar) very high adherence rates are achieved with all the materials tested, except with pink granite type 3 .

-The data obtained do not allow to determine which type of stone is more efficient, we recommend to carry out this test in every particular case.

-There's no relation between the adherences rates and the absortion values of the tested stones.

\section{THANKS TO}

The authours would like to express theirgratitude to the company VORSEVI S.A. without which we couldn't have carried out this study, and also to the Spanish Ministry of Education and Science for their statal Aid included in their Exchange Programme for Research.

\section{BIBLIOGRAFÍA}

(1) CSIC. Alteración de granitos y rocas afines. Ed. CSIC. 1993

(2) DE BENITO FERNÁNDEZ, J; GALINDO GARCÍA, P.; y OTROS. Curso de Rehabilitación. Cerramientos y Acabados. Ed. Servicio de Publicaciones del Colegio Oficial de Arquitectos de Madrid. 1988. 
(3) DE CUSA, J.; Colocación de alicatados. Ed. CEAC. 1993.

(4) IGME. Simposio sobre Rocas Ornamentales y Minerales Industriales. Ed. IGME. 1987.

(5) ITGE. Granitos de España. Ed. ITGE. 1991.

(6) ITGE. Mármoles de España. Ed. ITGE. 1991.

(7) MONJO CARRIÓN, J.; MALDONADO BARRIOS, L.; DELIBES LINIERS, A.; y OTROS.Curso de Patología. Conservación y restauración de edificios. Tomo l. Ed. Servicio de Publicaciones del Colegio Oficial de Arquitectos de Madrid. 1991.

(8) NTE Chapados. (RPC)

(9) PORCAR, J.L.; y COL. Manual-Guia Técnica de los revestimientos y pavimentos cerámicos. Ed. Instituto de Tecnología Cerámica Diputación de Castellón. 1987.

(10) UNE 83.822/92 (Experimental).

\section{publicaciones del ICCET/CSIC}

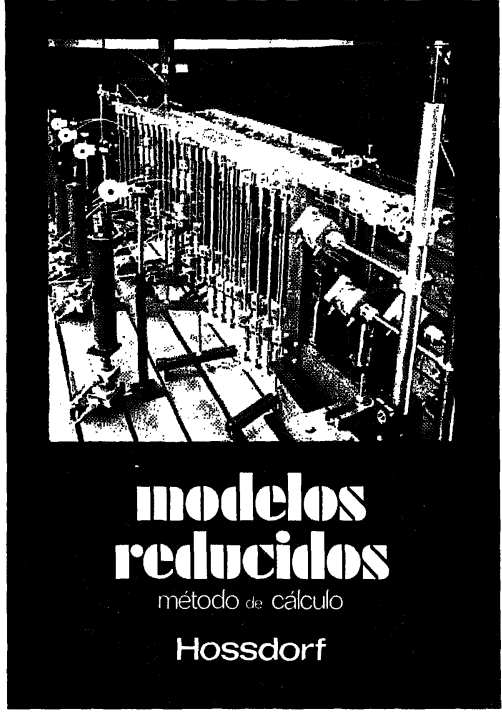

Modelos reducidos. Método de cálculo

H. Hossdorf, Ingeniero Civil

La técnica de los ensayos en modelos reducidos de estructuras sufre hoy dia una decisiva metamorfosis. Hasta hace poco era un medio más bien de artesania, que no siempre era tomado en serio por los nia, aue no siempre un medio mas oen ae aro académicos teorizantes Dara comprender el comportaque se acudió las más de las veces, como a un ultimo remedio debido a sus indiscutibles insuficien cias. Sin embargo, on a sus tiempo y gracias a su cias. Sin embargo, en poco tiempo y gracias a su conexión con los ordenadores digitales, se ha transque no puede quedar a un lado en la práctica diaria del Ingeniero Proyectista.

Un volumen encuadernado en cartoné plastificado 250 páginas, 158 figuras y fotografias.

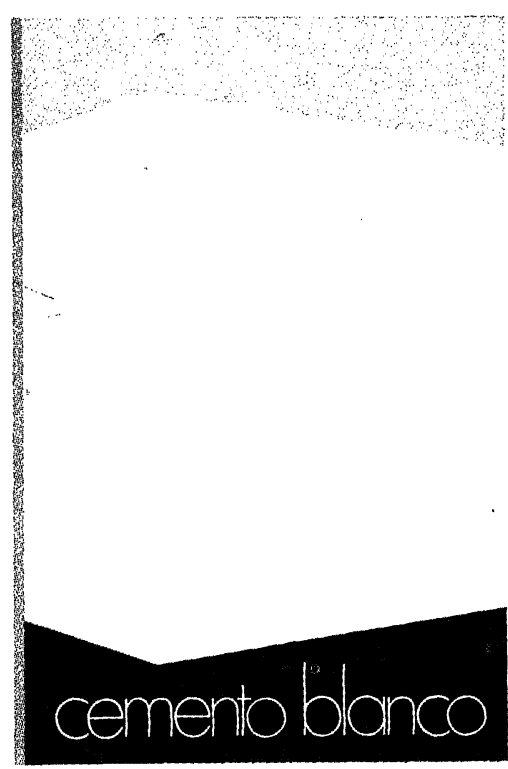

Cemento blanco

Julián Rezola

Ingeniero Quimico Dipl. I. Q. S

Sabido es que existe una extensa y documentada bibliografia sobre el cemento gris: en cambio, no puede decirse lo mismo acerca del cemento portiand blanco, ya que los escritos existentes se refieren tan sólo a algunas peculiaridades que le distinguen

El autor nos ofrece sus profundos conocimientos y su larga experiencia tanto en laboratorio como en fabricación.

La parte descriptiva del libro se complementa con gráficos, diagramas y fotografias de gran utilidad, destinados a conseguir la aplicación apropiada de este aglomerante.

Un volumen encuadernado en cartoné policerado, do $17,4 \times 24,3 \mathrm{~cm}$, compuesto de 395 pácginas, numerosas figuras, tablas $y$ ábacos.

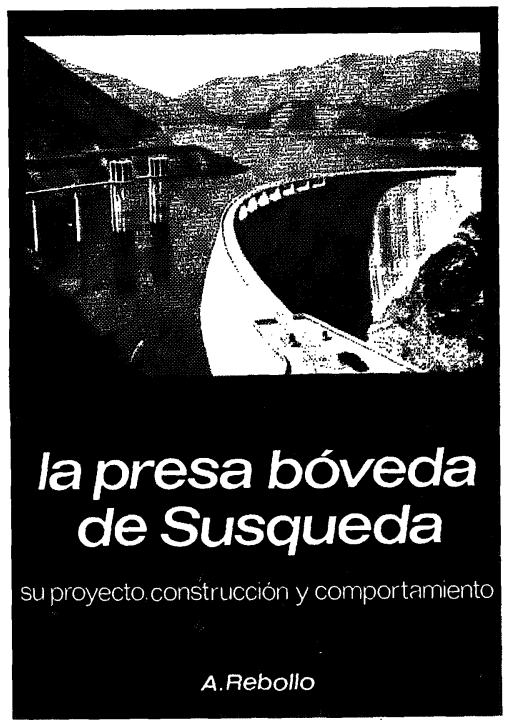

La presa bóveda de Susqueda

A. Rebollo

Dr. Ingeniero de Caminos

El esfuerzo del constructor de presas se sitúa por su pretensión de perennidad, a contracorriente de las tendencias de la civilización actual, caracte rizada por to fungible. Pueden evocarse las 10.000 grandes presas en funcionamiento o en construcción que estan envejeciendo y reclaman los cuidados gerontológicos para mantener y perfeccionar su servicio y garantizar su inalienable pretension de perennidad. En la medida en que todas nuevas obras, grandes o pequeñas, son portadoras de resgos ecologicos $y$, a veces, catastrolicos, que las presas cos el envejeciniento, la gerontogia de de Arsur es todo un entaro. La accion adelantada a seguir para todos los clue aman su propia obra co a devocion parernal que el ha puesto en Susqueda.

Un volumen encuadernado en cartoné plastificado con lomo de tela, de $18 \times 24,5 \mathrm{~cm}$, compuesto de 408 páginas, 330 figuras y fotografias y 39 tablas. 